\title{
Article \\ Boundary Conditions that Remove Certain Ultraviolet Divergences
}

\author{
Roderich Tumulka
}

check for

updates

Citation: Tumulka, R. Boundary Conditions that Remove Certain Ultraviolet Divergences. Symmetry 2021, 13, 577. https://doi.org/ 10.3390/sym13040577

Academic Editors: Nicola Maggiore and Ignatios Antoniadis

Received: 9 January 2021

Accepted: 23 March 2021

Published: 31 March 2021

Publisher's Note: MDPI stays neutral with regard to jurisdictional claims in published maps and institutional affiliations.

Copyright: (C) 2021 by the author. Licensee MDPI, Basel, Switzerland. This article is an open access article distributed under the terms and conditions of the Creative Commons Attribution (CC BY) license (https:// creativecommons.org/licenses/by/ $4.0 /)$.
Mathematisches Institut, Eberhard-Karls-Universität, Auf der Morgenstelle 10, 72076 Tübingen, Germany; roderich.tumulka@uni-tuebingen.de

\begin{abstract}
In quantum field theory, Hamiltonians contain particle creation and annihilation terms that are usually ultraviolet (UV) divergent. It is well known that these divergences can sometimes be removed by adding counter-terms and by taking limits in which a UV cutoff tends toward infinity. Here, I review a novel way of removing UV divergences: by imposing a type of boundary condition on the wave function. These conditions, called interior-boundary conditions (IBCs), relate the values of the wave function at two configurations linked by the creation or annihilation of a particle. They allow for a direct definition of the Hamiltonian without renormalization or limiting procedures. In the last section, I review another boundary condition that serves to determine the probability distribution of detection times and places on a time-like 3-surface.
\end{abstract}

Keywords: interior-boundary condition; particle creation; renormalization

\section{Introduction}

Let us take an unusual approach to quantum field theory (QFT): let us use wave functions. Specifically, let us use a particle-position representation of the quantum state vector. I know there are reservations about such an approach, but let us set aside these reservations for a little while and explore what this may gain us. My message here is that this approach has led to a certain new type of boundary conditions on the wave function, called interior-boundary conditions (IBCs), that make UV-finite particle creation and annihilation terms in the Hamiltonian (in some models, at least) possible.

Is it desirable to obtain a Hamiltonian without UV divergence? Many researchers have given up on this aim long ago and understandably so. If there has been not much progress on the UV divergence problem in a long time, then we will want to move on without solving this problem. However, that does not mean that a solution to the problem would not be welcome. Likewise, many physicists have focused on computing scattering matrices, which represent the time evolution from $t=-\infty$ to $t=+\infty$. However, that does not mean that the time evolution in between, for finite times, did not exist in nature. For those QFTs for which a well-defined Hamiltonian exists, it seems to me very valuable to know that it does.

In this paper, I explain the idea of IBCs and how it works. I report about some recent progress on providing well-defined Hamiltonians by means of IBCs, mainly concerning nonrelativistic models, and about some reasons for thinking that IBCs can also be applied in quantum electrodynamics (QED). It has turned out that Hamiltonians defined with IBCs agree with those obtained through renormalization in the cases where both approaches are available. However, the IBC approach has also been applied successfully to models for which no well-defined Hamiltonian was known (neither through renormalization nor otherwise). In the last section of this paper, I briefly turn to another boundary condition on wave functions relevant to the probability distribution of arrival times or, more precisely, detection times. 
In addition to UV divergence, some QFTs also have infrared (IR) divergence problems. However, they seem less serious because they usually disappear if 3 space is assumed to have finite volume, and it seems like a real possibility that this is the case in our universe.

IBCs were first considered by Moshinsky in 1951 [1-3] for the purpose of modeling particle creation and annihilation but not for dealing with UV divergence. This use was proposed in 2015 [4] and has led to a number of papers on IBCs since.

In Section 2, I discuss IBCs in the nonrelativistic case. I include an overview of the literature in Section 2.6 that also covers works on the relativistic case. In Section 3, I explain why there is reason to think that the approach can also be applied to QED and what the present obstacles are. In Section 4, I describe another boundary condition, an absorbing boundary condition, used for formulating Born's rule for a time-like 3-surface.

\section{Nonrelativistic Case}

Let us consider wave functions in the particle-position representation. In N-particle quantum mechanics, the wave function can be regarded as a function $\psi$ on configuration space $\mathbb{R}^{3 N}$ with values in spin space $S^{\otimes N}$ with $S=\mathbb{C}^{2 s+1}$ the spin space of a single spin-s particle. For models involving particle creation, $\psi$ should be a function on the configuration space $\mathcal{Q}$ of a variable number of particles,

$$
\mathcal{Q}=\bigcup_{n=0}^{\infty}\left(\mathbb{R}^{3}\right)^{n}
$$

We could use ordered configurations $\left(x_{1}, \ldots, x_{n}\right)$ or unordered configurations $\left\{x_{1}, \ldots, x_{n}\right\}$; it will be convenient for us to use ordered ones. On the $n$-particle sector of $\mathcal{Q}, \psi$ takes values in $S^{\otimes n}$, so it can be written as $\psi_{s_{1} \ldots s_{n}}\left(\boldsymbol{x}_{1} \ldots \boldsymbol{x}_{n}\right)$ with $s_{j} \in\{1, \ldots, 2 s+1\}$ and $\boldsymbol{x}_{j} \in \mathbb{R}^{3}$. For brevity, we often write $\left(x_{1} \ldots x_{n}\right)$ for $\left(x_{1}, \ldots, x_{n}\right)$. Furthermore, $\psi$ should be anti-symmetric (for fermions) or symmetric (for bosons) against permutations of the particle positions along with their spin indices. The set of (square-integrable) such wave functions $\psi$ is the (fermionic or bosonic) Fock space $\mathscr{F}$.

\subsection{Simple Model}

Let us consider two particle species, $x$ and $y$, such that $x$-particles (fermions) can emit and absorb $y$-particles (bosons), $x \rightleftarrows x+y$; this is sometimes called the Lee model [5,6]. For simplicity, let us focus first on the case of a single $x$-particle fixed at the origin $\mathbf{0} \in \mathbb{R}^{3}$; this is sometimes called the van Hove model [7,8]. For simplicity, let us suppose further that all particles, $x$ and $y$, have spin $s=0$ (although in nature all fermions have half-odd spin; spin-statistics theorems do not apply here, as the model is nonrelativistic). Then, $\psi$ is a complex-valued function of the $y$-configuration, $\psi=\psi\left(\boldsymbol{y}_{1} \ldots y_{n}\right)$ with variable particle number $n$, or equivalently a vector in the bosonic Fock space of the $y$-particles.

The natural formula for a Hamiltonian of free nonrelativistic $y$-particles that are created and annihilated at the origin is

$$
\begin{aligned}
\left(H_{\text {orig }} \psi\right)^{(n)}\left(\boldsymbol{y}_{1} \ldots \boldsymbol{y}_{n}\right)= & -\frac{\hbar^{2}}{2 m_{y}} \sum_{k=1}^{n} \nabla_{k}^{2} \psi^{(n)}\left(\boldsymbol{y}_{1} \ldots \boldsymbol{y}_{n}\right)+n E_{0} \psi^{(n)}\left(\boldsymbol{y}_{1} \ldots \boldsymbol{y}_{n}\right) \\
& +g \sqrt{n+1} \psi^{(n+1)}\left(\boldsymbol{y}_{1} \ldots \boldsymbol{y}_{n}, \mathbf{0}\right) \\
& +\frac{g}{\sqrt{n}} \sum_{k=1}^{n} \delta^{3}\left(\boldsymbol{y}_{k}\right) \psi^{(n-1)}\left(\boldsymbol{y}_{1} \ldots \boldsymbol{y}_{k-1}, \boldsymbol{y}_{k+1} \ldots \boldsymbol{y}_{n}\right)
\end{aligned}
$$

where $\psi^{(n)}$ means the $n$-particle sector of $\psi, m_{y}>0$ is the mass of a $y$-particle, $E_{0} \geq 0$ is the energy that needs to be expended for creating a $y$-particle (which would reasonably be $E_{0}=m_{y} c^{2}$, but that is not crucial for what follows), $g \in \mathbb{R}$ is a coupling constant governing the strength of the emission and absorption, and $\delta^{3}$ means the Dirac delta function in 3 dimensions. The first line represents the free time evolution of the $y \mathrm{~s}$, the second the 
absorption of $y_{n+1}$ by the $x$, and the third the creation of a $y$ by the $x$; the sum over $k$ ensures the permutation symmetry. In a different notation, the Hamiltonian can be written as

$$
H_{\text {orig }}=H_{\text {free }}+H_{\text {int }}
$$

with the free boson Hamiltonian

$$
H_{\text {free }}=d \Gamma\left(-\frac{\hbar^{2}}{2 m_{y}} \nabla^{2}+E_{0}\right)
$$

where $d \Gamma\left(H_{1}\right)$ means the "second quantization functor" applied to a 1-particle Hamiltonian $H_{1}$, and the interaction Hamiltonian

$$
H_{\text {int }}=g a(\mathbf{0})+g a^{\dagger}(\mathbf{0}),
$$

where $a(x)$ is the boson annihilation operator in the position representation at location $x$. In yet another notation,

$$
H_{\text {free }}=\int d^{3} \boldsymbol{k}\left(\frac{\hbar^{2} \boldsymbol{k}^{2}}{2 m_{y}}+E_{0}\right) a^{\dagger}(\boldsymbol{k}) a(\boldsymbol{k})
$$

and

$$
H_{\text {int }}=g \int \frac{d^{3} \boldsymbol{k}}{(2 \pi)^{3 / 2}} \phi(\boldsymbol{k})
$$

with $\phi(\boldsymbol{k})$ being the bosonic field operator for momentum $\hbar \boldsymbol{k}$, i.e.,

$$
\phi(\boldsymbol{k})=a^{\dagger}(\boldsymbol{k})+a(\boldsymbol{k})
$$

with $a(\boldsymbol{k})$ being the boson annihilation operator for momentum $\hbar \boldsymbol{k}$.

Now, for $g \neq 0, H_{\text {orig }}$ as in (2) or (3)-(7) is UV divergent-it does not actually define an operator in Fock space $\mathscr{F}$. Specifically, the integral over $\boldsymbol{k}$ in (7) diverges for large $|\boldsymbol{k}|$ (i.e., for "ultraviolet" $k$ ). Equivalently, one can say that the $\delta^{3}$ that appears as the wave function of the newly created $y$-particle is problematic; it can be thought of as containing an infinite amount of energy. Due to the $\delta^{3}$ factor, $H_{\text {orig }} \psi$ is not even a square-integrable function and thus does not lie in $\mathscr{F}$ even if $\psi$ is as nice a function as one could wish for, say, infinitely differentiable and compactly supported in each $\left(\mathbb{R}^{3}\right)^{n}$. Similar divergence problems have plagued most QFTs since the early days of quantum theory [9]. Over the next pages, I describe how, by means of boundary conditions on $\psi$, it is nevertheless possible to make sense of Equation (2) or Equations (3)-(7), that is, how a Hamiltonian can indeed be defined to describe the emission and absorption of particles at a single point $\mathbf{0}$.

Here, by the "boundary" of the $n$-particle sector $\mathcal{Q}^{(n)}=\left(\mathbb{R}^{3}\right)^{n}$, we mean the set

$$
\partial \mathcal{Q}^{(n)}=\left\{\left(\boldsymbol{y}_{1} \ldots \boldsymbol{y}_{n}\right) \in \mathcal{Q}^{(n)}: \boldsymbol{y}_{k}=\mathbf{0} \text { for some } k\right\}
$$

of configurations for which at least one $y$-particle meets the $x$-particle. Correspondingly, by the boundary of $\mathcal{Q}$, we mean

$$
\partial \mathcal{Q}=\bigcup_{n=0}^{\infty} \partial \mathcal{Q}^{(n)} .
$$

The relevant boundary condition is essentially a relation between the values of $\psi$ at two configurations related through the emission or absorption of a $y$-particle, i.e., at $\left(\boldsymbol{y}_{1} \ldots \boldsymbol{y}_{n}\right) \in$ $\mathcal{Q}^{(n)}$ and $\left(\boldsymbol{y}_{1} \ldots \boldsymbol{y}_{n}, \mathbf{0}\right) \in \partial \mathcal{Q}^{(n+1)}$ or a permutation thereof. Since the latter point lies on the boundary and the former does not, it is called an interior-boundary condition (IBC). The condition is essentially of the form

$$
\psi^{(n+1)}\left(\boldsymbol{y}_{1} \ldots \boldsymbol{y}_{n}, \mathbf{0}\right)=\alpha_{n} \psi^{(n)}\left(\boldsymbol{y}_{1} \ldots \boldsymbol{y}_{n}\right)
$$


with some fixed constant $\alpha_{n} \in \mathbb{R}$. The exact condition, Equation (29) below, will be a little more involved as the left-hand side needs to be replaced with the leading coefficient in the asymptotics when approaching $\left(\boldsymbol{y}_{1} \ldots \boldsymbol{y}_{n}, \mathbf{0}\right)$.

\subsection{Motivation: Probability Transport}

Before I write down the condition in Section 2.3, I give a motivation based on consideration of probability transport. As in $n$-particle quantum mechanics, $\left|\psi\left(\boldsymbol{y}_{1} \ldots y_{n}\right)\right|^{2}$ yields the probability density $\rho\left(\boldsymbol{y}_{1} \cdots \boldsymbol{y}_{n}\right)$, relative to the $3 n$-dimensional volume $d^{3} \boldsymbol{y}_{1} \cdots d^{3} \boldsymbol{y}_{n}$, of the configuration being at $\left(\boldsymbol{y}_{1} \ldots y_{n}\right)$. Since this is a probability density on $\mathcal{Q}$, its integral over $\mathcal{Q}$ will equal unity,

$$
\int_{\mathcal{Q}} d y|\psi(y)|^{2}=\sum_{n=0}^{\infty} \int_{\mathbb{R}^{3 n}} d^{3} \boldsymbol{y}_{1} \cdots d^{3} \boldsymbol{y}_{n}\left|\psi\left(\boldsymbol{y}_{1} \cdots \boldsymbol{y}_{n}\right)\right|^{2}=1 .
$$

Additionally, as in quantum mechanics, the transport of probability within each sector $\mathcal{Q}^{(n)}=\mathbb{R}^{3 n}$ is governed by the probability current $j$, which is a vector field on $\mathcal{Q}$ given on any sector $\mathbb{R}^{3 n}$ by

$$
j=\frac{\hbar}{m_{y}} \operatorname{Im}\left[\psi^{*} \nabla \psi\right] .
$$

However, while in quantum mechanics of $N$ particles, $\rho$ is related to $j$ by the continuity equation

$$
\frac{\partial \rho}{\partial t}=-\nabla \cdot j
$$

in $\mathbb{R}^{3 N}$, the equation in $\mathcal{Q}$ that looks the same as (14) cannot be expected to be valid: after all, $j$ only represents probability transport within one sector and thus cannot account for probability transport from one sector to another as occurs through particle creation or annihilation. Since particle creation transports probability from whichever configuration $\left(\boldsymbol{y}_{1} \ldots \boldsymbol{y}_{n}\right)$ to another configuration $\left(\boldsymbol{y}_{1} \ldots \boldsymbol{y}_{n}, \mathbf{0}\right)$ (or a permutation thereof), the amount of probability from $\mathcal{Q}^{(n)}$ when transported to $\mathcal{Q}^{(n+1)}$ will show up at $\partial \mathcal{Q}^{(n+1)}$. Conversely, amounts of probability can be transported from $\mathcal{Q}^{(n+1)}$ to $\mathcal{Q}^{(n)}$ through annihilation, in which case, they are transported from $\left(\boldsymbol{y}_{1} \ldots \boldsymbol{y}_{n}, \mathbf{0}\right)$ (or a permutation thereof) to $\left(\boldsymbol{y}_{1} \ldots \boldsymbol{y}_{n}\right)$, so that they can only be transported from $\partial \mathcal{Q}^{(n+1)}$ to $\mathcal{Q}^{(n)}$. Then, what is the equation in $\mathcal{Q}$ replacing (14)? At configurations $\left(\boldsymbol{y}_{1} \ldots \boldsymbol{y}_{n}\right)$ not on the boundary $\partial \mathcal{Q}$, it reads

$$
\frac{\partial \rho}{\partial t}=-\nabla \cdot j-J
$$

with $J\left(\boldsymbol{y}_{1} \ldots \boldsymbol{y}_{n}\right)$ being the probability current coming out of $\partial \mathcal{Q}^{(n+1)}$ at $\left(\boldsymbol{y}_{1} \ldots \boldsymbol{y}_{n}, \mathbf{0}\right)$ (and permutations thereof) minus the probability current flowing into $\partial \mathcal{Q}^{(n+1)}$ at $\left(\boldsymbol{y}_{1} \ldots \boldsymbol{y}_{n}, \mathbf{0}\right)$ (and permutations thereof). These currents can be expressed as flux integrals of $j$. To see how, let us begin with a simple example: for a current vector field $j$ in $\mathbb{R}^{3}$, the flux out of the sphere of radius $r$ minus the flux into it is given by

$$
\int_{\mathbb{S}^{2}} d^{2} \boldsymbol{\omega} r^{2} \boldsymbol{\omega} \cdot j(r \boldsymbol{\omega})
$$

where $\mathbb{S}^{2}$ denotes the unit sphere in $\mathbb{R}^{3}$ and $d^{2} \boldsymbol{\omega}$ is the area of a surface element of the sphere. Note that $\omega \cdot j$ is the radial component of $j$, which is positive for an outward current and negative for an inward current. Taking the limit $r \searrow 0$, we obtain the flux out of the origin minus the flux into it. Correspondingly, $J$, the flux out of (permutations of) $\left(y_{1} \ldots y_{n}, 0\right)$ minus the flux into it, is given by

$$
J\left(\boldsymbol{y}_{1} \ldots \boldsymbol{y}_{n}\right)=\sum_{k=1}^{n+1} \lim _{r \searrow 0} \int_{\mathbb{S}^{2}} d^{2} \boldsymbol{\omega} r^{2} \boldsymbol{\omega} \cdot \boldsymbol{j}_{k}\left(\boldsymbol{y}_{1} \ldots \boldsymbol{y}_{k-1}, r \boldsymbol{\omega}, \boldsymbol{y}_{k} \ldots \boldsymbol{y}_{n}\right),
$$


where $j_{k}$ means the 3 particle- $k$ components of $j$, i.e., $j=\left(j_{1} \ldots j_{n+1}\right)$. Note that $r \omega$ does not replace $\boldsymbol{y}_{k}$ but gets inserted between $\boldsymbol{y}_{k-1}$ and $\boldsymbol{y}_{k^{\prime}}$, so that $j_{k}$ has $n+1$ arguments and, for $k=n+1$, equals $j_{n+1}\left(y_{1} \ldots y_{n}, r \omega\right)$.

For the expression (17) to be nonzero, we need $\omega \cdot j_{k}$ to be large or else the factor $r^{2}$ in the integral will push the integral to 0 in the limit; that is, $\omega \cdot j_{k}$ should be large like $1 / r^{2}$. Since $j$ is quadratic in $\psi, \psi$ should be large like $1 / r$ near $\partial \mathcal{Q}$.

Now that we know what the needed continuity equation (15) looks like, we ask what the Hamiltonian should look like in order to entail this continuity equation. Writing

$$
H=H_{\text {free }}+H_{\text {int }}
$$

with $H_{\text {free }}=d \Gamma\left(-\frac{\hbar^{2}}{2 m_{y}} \nabla^{2}+E_{0}\right)$ (i.e., acting like the first line of (2)), it follows from Schrödinger's equation

$$
i \hbar \frac{\partial \psi}{\partial t}=H \psi
$$

that

$$
\begin{aligned}
\frac{\partial|\psi|^{2}}{\partial t} & =\frac{2}{\hbar} \operatorname{Im}\left[\psi^{*}(H \psi)\right] \\
& =\frac{2}{\hbar} \operatorname{Im}\left[\psi^{*}\left(H_{\text {free }} \psi\right)\right]+\frac{2}{\hbar} \operatorname{Im}\left[\psi^{*}\left(H_{\text {int }} \psi\right)\right] .
\end{aligned}
$$

Since

we obtain that

$$
\frac{2}{\hbar} \operatorname{Im}\left[\psi^{*}\left(H_{\text {free }} \psi\right)\right]=-\nabla \cdot j,
$$

$$
\frac{\partial \rho}{\partial t}=-\nabla \cdot j+\frac{2}{\hbar} \operatorname{Im}\left[\psi^{*}\left(H_{\text {int }} \psi\right)\right],
$$

and in order to reach the desired form (15), we need that

$$
\begin{aligned}
& \stackrel{2}{\hbar} \operatorname{Im}\left[\psi^{(n)}\left(\boldsymbol{y}_{1} \ldots \boldsymbol{y}_{n}\right)^{*}\left(H_{\mathrm{int}} \psi\right)^{(n)}\left(\boldsymbol{y}_{1} \ldots \boldsymbol{y}_{n}\right)\right] \\
& \quad=-J\left(\boldsymbol{y}_{1} \ldots \boldsymbol{y}_{n}\right) \\
& \quad \stackrel{(17)}{=}-(n+1) \lim _{r \searrow 0} \int_{\mathbb{S}^{2}} d^{2} \boldsymbol{\omega} r^{2} \boldsymbol{\omega} \cdot \boldsymbol{j}_{n+1}\left(\boldsymbol{y}_{1} \ldots \boldsymbol{y}_{n}, r \boldsymbol{\omega}\right) \\
& \quad \stackrel{(13)}{=}-(n+1) \lim _{r \searrow 0} \int_{\mathbb{S}^{2}} d^{2} \boldsymbol{\omega} r^{2} \frac{\hbar}{m_{y}} \operatorname{Im}\left[\psi^{(n+1)}\left(\boldsymbol{y}_{1} \ldots y_{n}, r \boldsymbol{\omega}\right)^{*} \partial_{r} \psi^{(n+1)}\left(\boldsymbol{y}_{1} \ldots y_{n}, r \boldsymbol{\omega}\right)\right] \\
& =-\frac{\hbar}{m_{y}}(n+1) \operatorname{Im}\left[\lim _{r \searrow 0} \int_{\mathbb{S}^{2}} d^{2} \boldsymbol{\omega} r \psi^{(n+1)}\left(\boldsymbol{y}_{1} \ldots y_{n}, r \boldsymbol{\omega}\right)^{*} \partial_{r}(r \psi)^{(n+1)}\left(\boldsymbol{y}_{1} \ldots y_{n}, r \boldsymbol{\omega}\right)\right] .
\end{aligned}
$$

In step (25), we used that, by permutation symmetry of $\psi$, all $n+1$ summands of (17) are equal; in step (26), that $\omega \cdot \nabla \psi(r \omega)=\partial_{r} \psi(r \omega)$; and in step (27), that $\partial_{r}(r \psi)=\psi+r \partial_{r} \psi$, so that

$$
\operatorname{Im}\left[r \psi^{*} \partial_{r}(r \psi)\right]=\operatorname{Im}\left[r \psi^{*} \psi+r^{2} \psi^{*} \partial_{r} \psi\right]=\operatorname{Im}\left[r^{2} \psi^{*} \partial_{r} \psi\right] .
$$

Now, we compare the left-hand side of (24) to the right-hand side of (27). We have imaginary parts on both sides, so the equation would be fulfilled if the square brackets could be made to agree up to the appropriate pre-factors. $\left(H_{\text {int }} \psi\right)^{(n)}$ could certainly involve $\psi^{(n+1)}$, but the factors of $\psi^{*}$ seem more problematic. We would need that $r \psi^{(n+1)}\left(\boldsymbol{y}_{1} \ldots \boldsymbol{y}_{n}, r \boldsymbol{\omega}\right)$ is, in the limit $r \rightarrow 0$, proportional to $\psi^{(n)}\left(\boldsymbol{y}_{1} \ldots \boldsymbol{y}_{n}\right)$. We would need, in other words, a boundary condition on $\psi^{(n+1)}$. We can now easily guess what the IBC should say, except for the choice of the proportionality factor $\alpha_{n}$, which will become clear soon. 


\subsection{Interior-Boundary Condition}

Here is what the IBC says: For every $\boldsymbol{\omega} \in \mathbb{S}^{2}, n \in\{0,1,2, \ldots\}$, and $\boldsymbol{y}_{1} \ldots \boldsymbol{y}_{n} \in \mathbb{R}^{3} \backslash\{\mathbf{0}\}$,

$$
\lim _{r \searrow 0}\left(r \psi^{(n+1)}\left(\boldsymbol{y}_{1} \ldots \boldsymbol{y}_{n}, r \boldsymbol{\omega}\right)\right)=-\frac{g m_{y}}{2 \pi \hbar^{2} \sqrt{n+1}} \psi^{(n)}\left(\boldsymbol{y}_{1} \ldots \boldsymbol{y}_{n}\right) .
$$

The Hamiltonian $H=H_{\mathrm{IBC}}$ acts on wave functions satisfying this condition according to

$$
\begin{aligned}
\left(H_{\mathrm{IBC}} \psi\right)^{(n)}\left(\boldsymbol{y}_{1} \ldots \boldsymbol{y}_{n}\right)= & -\frac{\hbar^{2}}{2 m_{y}} \sum_{k=1}^{n} \nabla_{k}^{2} \psi^{(n)}\left(\boldsymbol{y}_{1} \ldots \boldsymbol{y}_{n}\right)+n E_{0} \psi^{(n)}\left(\boldsymbol{y}_{1} \ldots \boldsymbol{y}_{n}\right) \\
& +\frac{g \sqrt{n+1}}{4 \pi} \int_{\mathbb{S}^{2}} d^{2} \boldsymbol{\omega} \lim _{r \searrow 0} \frac{\partial}{\partial r}\left(r \psi^{(n+1)}\left(\boldsymbol{y}_{1} \ldots \boldsymbol{y}_{n}, r \boldsymbol{\omega}\right)\right) \\
& +\frac{g}{\sqrt{n}} \sum_{k=1}^{n} \delta^{3}\left(\boldsymbol{y}_{k}\right) \psi^{(n-1)}\left(\boldsymbol{y}_{1} \ldots \boldsymbol{y}_{k-1}, \boldsymbol{y}_{k+1} \ldots \boldsymbol{y}_{n}\right) .
\end{aligned}
$$

It is now easy to check, by inserting the IBC (29) into (27), that

$$
\begin{aligned}
(27) & =\frac{g}{2 \pi \hbar} \sqrt{n+1} \operatorname{Im}\left[\lim _{r \searrow 0} \int_{\mathbb{S}^{2}} d^{2} \boldsymbol{\omega} \psi^{(n)}\left(\boldsymbol{y}_{1} \ldots \boldsymbol{y}_{n}\right)^{*} \partial_{r}(r \psi)^{(n+1)}\left(\boldsymbol{y}_{1} \ldots \boldsymbol{y}_{n}, r \boldsymbol{\omega}\right)\right] \\
& =\frac{2}{\hbar} \operatorname{Im}\left[\psi^{(n)}\left(\boldsymbol{y}_{1} \ldots \boldsymbol{y}_{n}\right)^{*} \frac{g \sqrt{n+1}}{4 \pi} \int_{\mathbb{S}^{2}} d^{2} \boldsymbol{\omega} \lim _{r \searrow 0} \partial_{r}(r \psi)^{(n+1)}\left(\boldsymbol{y}_{1} \ldots \boldsymbol{y}_{n}, r \boldsymbol{\omega}\right)\right],
\end{aligned}
$$

in agreement with the left-hand side of (24) and (30) at a configuration not on the boundary.

Equations (29) and (30) form the solution to the UV divergence problem that I want to present:

Theorem 1 ([10]). Equation (30) provides a well-defined, self-adjoint operator $H_{\mathrm{IBC}}$ on a dense domain in Fock space $\mathscr{F}$ containing wave functions $\psi$ that satisfy the IBC (29). The operator is bounded from below.

This means that the UV divergence is absent from $H_{\mathrm{IBC}}$. Additionally, I argue below that $H_{\mathrm{IBC}}$ is a reasonable interpretation of the original expression $H_{\text {orig }}$ of (2).

Sometimes in QFT, one uses boundary conditions on 1-particle propagators, i.e., on Green's functions. The boundary condition (29) is not like that. For one thing, it is a condition on the wave function, but more importantly, it is not a 1-particle condition but instead links the $n$-particle sector with the $n+1$-particle sector.

\subsection{Why It Works and Why It Is Reasonable}

Two things may seem surprising: First, how the divergence problem can be absent if the $\delta^{3}$ that was the root of the trouble is still present and, second, why anyone would regard the middle line of (30) as a reasonable interpretation of the middle line of (2).

Here is why $\delta^{3}$ does not cause trouble any more: Note that $\psi^{(n)}$ diverges at the boundary $\partial \mathcal{Q}^{(n)}$ like $1 / r$, with $r$ being the distance from the boundary,

$$
\psi^{(n)}\left(\boldsymbol{y}_{1} \ldots \boldsymbol{y}_{n-1}, r \boldsymbol{\omega}\right) \approx c r^{-1}
$$

with a complex coefficient $c$ that depends on $\boldsymbol{y}_{1}, \ldots, \boldsymbol{y}_{n-1}$. Note further that the Laplacian of $1 / r$ is a delta function,

$$
\nabla_{y}^{2} \frac{1}{|\boldsymbol{y}|}=-4 \pi \delta^{3}(\boldsymbol{y})
$$


a fact familiar from electrostatics, where the potential $\phi$ obeys the Poisson equation $\nabla^{2} \phi=-4 \pi \rho$ with the charge density $\rho$, and the potential of a point charge $\rho=\delta^{3}$ is the Coulomb potential $1 / r$. As a consequence, the Laplacian of $\psi^{(n)}$ will contain $\delta^{3}$ contributions, and they cancel exactly the $\delta^{3}$ contributions in the definition (30) of $H_{\mathrm{IBC}}$, with the effect that $H_{\mathrm{IBC}} \psi$ is actually a square-integrable function and thus lies in $\mathscr{F}$. Indeed, putting (33) and (34) together, we obtain that

$$
-\frac{\hbar^{2}}{2 m_{y}} \nabla_{n}^{2} \psi^{(n)}\left(\boldsymbol{y}_{1} \ldots \boldsymbol{y}_{n}\right)=c \frac{4 \pi \hbar^{2}}{2 m_{y}} \delta^{3}\left(\boldsymbol{y}_{n}\right)+\text { a function, }
$$

where we separated the singular (distributional) part (that involves a Dirac delta) from the regular part (that is a function). By virtue of the IBC (29),

$$
c=-\frac{g m_{y}}{2 \pi \hbar^{2} \sqrt{n}} \psi^{(n-1)}\left(\boldsymbol{y}_{1} \ldots \boldsymbol{y}_{n-1}\right),
$$

so

$$
-\frac{\hbar^{2}}{2 m_{y}} \nabla_{n}^{2} \psi^{(n)}\left(\boldsymbol{y}_{1} \ldots \boldsymbol{y}_{n}\right)=-\frac{g}{\sqrt{n}} \delta^{3}\left(\boldsymbol{y}_{n}\right) \psi^{(n-1)}\left(\boldsymbol{y}_{1} \ldots \boldsymbol{y}_{n-1}\right)+\text { a function, }
$$

which cancels exactly the $\delta^{3}$ in the $n$th summand of the last line of (30).

Let us turn to a comparison between the middle line of (30) and that of (2). Suppose for a moment (I will relax this supposition by the end of the subsection) that $\psi^{(n+1)}\left(\boldsymbol{y}_{1} \ldots \boldsymbol{y}_{n}, r \boldsymbol{\omega}\right)$ can be expanded into powers of $r$,

$$
\psi^{(n+1)}\left(\boldsymbol{y}_{1} \ldots \boldsymbol{y}_{n}, r \boldsymbol{\omega}\right)=\sum_{\ell=-1}^{\infty} c_{\ell} r^{\ell}
$$

starting at exponent -1 , with complex coefficients $c_{\ell}$ that may depend on $y_{1}, \ldots, y_{n}$ and $\omega$. Then,

$$
\begin{aligned}
\partial_{r}\left(r \psi^{(n+1)}\left(\boldsymbol{y}_{1} \ldots \boldsymbol{y}_{n}, r \boldsymbol{\omega}\right)\right) & =\partial_{r}\left(r \sum_{\ell=-1}^{\infty} c_{\ell} r^{\ell}\right) \\
& =\partial_{r} \sum_{\ell=-1}^{\infty} c_{\ell} r^{\ell+1} \\
& =\sum_{\ell=0}^{\infty} c_{\ell}(\ell+1) r^{\ell}
\end{aligned}
$$

starting at exponent 0 , and taking $r \rightarrow 0$, only the $\ell=0$ term remains, yielding $c_{0}$. Thus, the middle line of $H_{\mathrm{IBC}}$ in (30) equals

$$
\frac{g \sqrt{n+1}}{4 \pi} \int_{\mathbb{S}^{2}} d^{2} \boldsymbol{\omega} c_{0}\left(\boldsymbol{y}_{1} \ldots y_{n}, \boldsymbol{\omega}\right) .
$$

For comparison, the middle line of $H_{\text {orig }}$ in (2) asks us to evaluate $\psi^{(n+1)}$ at $\left(\boldsymbol{y}_{1} \ldots \boldsymbol{y}_{n}, \mathbf{0}\right)$; since $\psi^{(n+1)}$ diverges there, reasonable interpretations could be either to take the leading coefficient $c_{-1}$ or to use the nondivergent part $\sum_{\ell=0}^{\infty} c_{\ell} r^{\ell}$ and to evaluate that at $r=0$, which yields $c_{0}$. Since the IBC (29) fixes $c_{-1}$, as it demands that

$$
c_{-1}\left(\boldsymbol{y}_{1} \ldots \boldsymbol{y}_{n}, \boldsymbol{\omega}\right)=-\frac{g m_{y}}{2 \pi \hbar^{2} \sqrt{n+1}} \psi^{(n)}\left(\boldsymbol{y}_{1} \ldots \boldsymbol{y}_{n}\right),
$$

this first interpretation would not actually couple $H \psi^{(n)}$ to $\psi^{(n+1)}$ and therefore would not work. That leaves us with the second interpretation, according to which the middle line of (2) means $g \sqrt{n+1} c_{0}\left(y_{1} \ldots y_{n}, \boldsymbol{w}\right)$. Here, the question arises which $\boldsymbol{\omega}$ to use, and an obvious choice is to average over all $\omega \mathrm{s}$, which leads us to (42), which coincides with the 
middle line of $H_{\mathrm{IBC}}$ in (30). By the way, it turns out that, for $\psi$ in the domain of $H_{\mathrm{IBC}}$, both $c_{-1}$ and $c_{0}$ are actually independent of $\omega$ (or else $\nabla^{2} \psi$ would not be square-integrable away from the boundary; see ([11] Sec. 3.3 Rem. 7)), so that averaging over $\omega$ becomes unnecessary.

As a technical remark, we can drop the supposition that $\psi^{(n+1)}\left(\boldsymbol{y}_{1} \ldots \boldsymbol{y}_{n}, \boldsymbol{r} \boldsymbol{\omega}\right)$ can be expanded into powers of $r$ as in (38). As proven in [10], every $\psi$ in the domain of $H_{\mathrm{IBC}}$ is, as $r \rightarrow 0$, of the asymptotic form

$$
\psi^{(n+1)}\left(\boldsymbol{y}_{1} \ldots \boldsymbol{y}_{n}, r \boldsymbol{\omega}\right)=c_{-1}\left(\boldsymbol{y}_{1} \ldots y_{n}\right) r^{-1}+f\left(\boldsymbol{y}_{1} \ldots \boldsymbol{y}_{n}, r \boldsymbol{\omega}\right)
$$

with a function $f$ from the second Sobolev space; for such an $f$, both $f$ and $\partial_{r} f$ possess a finite limit as $r \rightarrow 0$, so that

$$
\lim _{r \rightarrow 0} \partial_{r}\left(r \psi^{(n+1)}\left(\boldsymbol{y}_{1} \ldots \boldsymbol{y}_{n}, r \boldsymbol{\omega}\right)\right)=f\left(\boldsymbol{y}_{1} \ldots \boldsymbol{y}_{n}, \mathbf{0}\right),
$$

which is the analog of the $c_{0}\left(y_{1} \ldots y_{n}\right)$ mentioned before and leads us again to the conclusion that the middle line of $H_{\mathrm{IBC}}$ in (30) agrees with a reasonable interpretation of the middle line of $H_{\text {orig }}$ in (2).

\subsection{Comparison to Renormalization}

Another argument to the effect that $H_{\mathrm{IBC}}$ is a reasonable interpretation of $H_{\text {orig }}$ comes from renormalization. Here, renormalization means to introduce a UV cutoff into the Hamiltonian and to then take a limit of removing the cutoff, possibly after adding a counterterm, in order to obtain a limiting Hamiltonian $H_{\infty}$ called the renormalized Hamiltonian. For the model discussed above, it has long been known that such a limit $H_{\infty}$ exists $[7,8]$, and it has recently turned out [10] that $H_{\infty}$ agrees with $H_{\mathrm{IBC}}$ up to addition of a constant (i.e., of a multiple of the identity),

$$
H_{\infty}=H_{\mathrm{IBC}}+E
$$

for some $E \in \mathbb{R}$. Needless to say, Hamiltonians that differ only by the addition of a constant $E, H^{\prime}=H+E$, are usually regarded as physically equivalent because the time-evolved state vectors they generate differ only by a global phase factor,

$$
e^{-i H^{\prime} t / \hbar} \psi_{0}=e^{-i E t / \hbar} e^{-i H t / \hbar} \psi_{0}
$$

and are thus considered physically equivalent. The fact that two different approaches to obtaining a well-defined Hamiltonian from the expression $H_{\text {orig }}$ lead to the same result suggests that this result is indeed a reasonable, physically appropriate interpretation of $H_{\text {orig }}$.

In more detail, to introduce a cutoff here means to replace the delta distribution $\delta^{3}$ in $H_{\text {orig }}$ by a regular function $\varphi: \mathbb{R}^{3} \rightarrow \mathbb{R}$ approximating it. We may assume that $\varphi$ is smooth, compactly supported, square-integrable, and perhaps rotationally symmetric. The cutoff Hamiltonian is then given by

$$
\begin{aligned}
\left(H_{\varphi} \psi\right)^{(n)}\left(\boldsymbol{y}_{1} \ldots \boldsymbol{y}_{n}\right)= & -\frac{\hbar^{2}}{2 m_{y}} \sum_{k=1}^{n} \nabla_{k}^{2} \psi^{(n)}\left(\boldsymbol{y}_{1} \ldots \boldsymbol{y}_{n}\right)+n E_{0} \psi^{(n)}\left(\boldsymbol{y}_{1} \ldots \boldsymbol{y}_{n}\right) \\
& +g \sqrt{n+1} \int_{\mathbb{R}^{3}} d^{3} \boldsymbol{y} \varphi(\boldsymbol{y}) \psi^{(n+1)}\left(\boldsymbol{y}_{1} \ldots \boldsymbol{y}_{n}, \boldsymbol{y}\right) \\
& +\frac{g}{\sqrt{n}} \sum_{k=1}^{n} \varphi\left(\boldsymbol{y}_{k}\right) \psi^{(n-1)}\left(\boldsymbol{y}_{1} \ldots \boldsymbol{y}_{k-1}, \boldsymbol{y}_{k+1} \ldots \boldsymbol{y}_{n}\right) .
\end{aligned}
$$

where $\delta^{3}$ was replaced by $\varphi$ in the last line and evaluation at 0 was replaced by the inner product with $\varphi$ in the last variable in the middle line. $H_{\varphi}$ is known (e.g., [7]) to be well- 
defined and self-adjoint. One may think of $\varphi$ as a continuous charge distribution, with the effect that the $x$-particle is no longer a point particle but spread out and can emit and absorb $y$-particles not only at the origin 0 but also in a whole neighborhood.

To remove the cutoff then means to take a limit in which $\varphi$ converges to $\delta^{3}$ (denoted $\varphi \rightarrow \delta^{3}$ ). As I will outline at the end of this subsection, it can be shown $[7,8,10]$ that, if the rest energy $E_{0}$ in (48) is positive, then there exists a self-adjoint operator $H_{\infty}$ and for every $\varphi$ a real number $E_{\varphi}$ such that $E_{\varphi} \rightarrow \infty$ and

$$
H_{\varphi}+E_{\varphi} \rightarrow H_{\infty} \text { as } \varphi \rightarrow \delta^{3} .
$$

Remarkably, although the existence of $H_{\infty}$ has long been known, it had not been realized until recently that the functions in the domain of $H_{\infty}$ satisfy the IBC (29) and that $H_{\infty}$ acts on them according to the formula (30) up to the addition of a constant.

The trick to taking the limit $\varphi \rightarrow \delta^{3}[7]$ is to write $H_{\varphi}$, which is

$$
H_{\varphi}=\int d^{3} \boldsymbol{k}\left(\omega(\boldsymbol{k}) a^{\dagger}(\boldsymbol{k}) a(\boldsymbol{k})+g \hat{\varphi}(\boldsymbol{k})^{*} a(\boldsymbol{k})+g \hat{\varphi}(\boldsymbol{k}) a^{\dagger}(\boldsymbol{k})\right)
$$

with $\omega(\boldsymbol{k})=\hbar^{2} \boldsymbol{k}^{2} / 2 m_{y}+E_{0}$, in the form of completing the square,

$$
H_{\varphi}=\int d^{3} \boldsymbol{k} \omega(\boldsymbol{k})\left(a^{\dagger}(\boldsymbol{k})+g \frac{\hat{\varphi}(\boldsymbol{k})^{*}}{\omega(\boldsymbol{k})}\right)\left(a(\boldsymbol{k})+g \frac{\hat{\varphi}(\boldsymbol{k})}{\omega(\boldsymbol{k})}\right)-\int d^{3} \boldsymbol{k} g^{2} \frac{|\hat{\varphi}(\boldsymbol{k})|^{2}}{\omega(\boldsymbol{k})},
$$

and to regard the last integral as $E_{\varphi}$. The unitary Bogolyubov transformation (dressing operator)

$$
W_{\varphi}:=\exp \left(\int d^{3} \boldsymbol{k} \frac{\hat{\varphi}(\boldsymbol{k})^{*}}{\omega(\boldsymbol{k})} a(\boldsymbol{k})-\int d^{3} \boldsymbol{k} \frac{\hat{\varphi}(\boldsymbol{k})}{\omega(\boldsymbol{k})} a^{\dagger}(\boldsymbol{k})\right)
$$

intertwines $H_{\varphi}+E_{\varphi}$ with the free Hamiltonian,

$$
H_{\varphi}+E_{\varphi}=W_{\varphi} H_{\text {free }} W_{\varphi}^{\dagger}
$$

and, for $E_{0}>0$, possesses a limit $W_{\varphi} \rightarrow W_{\infty}$ as $\hat{\varphi}(\boldsymbol{k}) \rightarrow$ const. or $\varphi \rightarrow \delta^{3}$, as already suggested by the fact that $1 / \omega(\boldsymbol{k})$ is still square integrable, so that the right-hand side of (52) is well defined when replacing $\hat{\varphi}$ with a real constant. Putting the pieces together, $H_{\varphi}+E_{\varphi} \rightarrow H_{\infty}=W_{\infty} H_{\text {free }} W_{\infty}^{\dagger}$.

As a future project, it would also be of interest to carry out a comparison of the IBC approach with the Epstein-Glaser approach $[12,13]$ for renormalization of the perturbation series for the scattering matrix.

\subsection{Other Models and Literature}

The mathematical analysis of IBC Hamiltonians is similar to that of point interaction (zero-range interaction) [14], i.e., potentials involving Dirac delta distributions as in

$$
H \psi(x)=-\nabla^{2} \psi+g \delta^{3}(x) \psi(x) .
$$

In fact, point interaction can also be expressed through a boundary condition on the wave function, the Bethe-Peierls boundary condition [15]

$$
\lim _{r \searrow 0}\left(\alpha+\frac{\partial}{\partial r}\right)(r \psi(r \omega))=0
$$

with a given constant $\alpha \in \mathbb{R}$.

The IBC (29) is not the only possible IBC for an $x$-particle fixed at the origin. Rather, there is a 4-parameter family of IBCs and associated self-adjoint Hamiltonians $[4,10,16,17]$, which can be thought of as having, in addition to the particle creation and annihilation at 
the origin, also a point interaction at the origin $[10,18]$. These Hamiltonians and IBCs can be characterized as follows. On wave functions that diverge on the boundary like $1 / r$, we can define two annihilation operators, $A$ and $B$, that extract from $\psi$ as in (38) the coefficient $c_{0}$ of order 0 and $c_{-1}$ of order -1 , respectively:

$$
A \psi^{(n)}\left(\boldsymbol{y}_{1} \ldots \boldsymbol{y}_{n}\right)=\sqrt{n+1} c_{0}\left(\boldsymbol{y}_{1} \ldots \boldsymbol{y}_{n}\right), \quad B \psi^{(n)}\left(\boldsymbol{y}_{1} \ldots \boldsymbol{y}_{n}\right)=\sqrt{n+1} c_{-1}\left(\boldsymbol{y}_{1} \ldots \boldsymbol{y}_{n}\right) .
$$

Then, the IBC (29) can be rewritten as

$$
B \psi=-\frac{g m_{y}}{2 \pi \hbar^{2}} \psi,
$$

and the action of the Hamiltonian $H(30)$ can be rewritten as

$$
H_{\mathrm{IBC}}=H_{\text {free }}+g A+g a^{\dagger}(\mathbf{0}) .
$$

Now, replacing $A \rightarrow e^{i \theta}(\alpha A+\beta B)$ and $B \rightarrow e^{i \theta}(\gamma A+\delta B)$ with real constants $\alpha, \beta, \gamma, \delta, \theta$ subject to $\alpha \delta-\beta \gamma=1$ yields further members of the 4-parameter family of IBCs and self-adjoint Hamiltonians ([10] Section 4). The families of possible IBCs for higher spins were identified in [19].

While the model of Sections 2.1-2.5 above assumes that the $x$-particle is fixed at the origin, a more realistic model assumes several moving $x$-particles. The Hilbert space is then of the form $\mathscr{H}=\mathscr{F}_{x} \otimes \mathscr{F}_{y}$ with $\mathscr{F}_{x}$ being a fermionic and $\mathscr{F}_{y}$ being a bosonic Fock space, and the configuration space is of the form $\mathcal{Q}_{x} \times \mathcal{Q}_{y}$ with each factor being a copy of the $\mathcal{Q}$ as in (1). The relevant boundary is then the set

$$
\partial \mathcal{Q}=\left\{\left(x_{1} \ldots x_{m}, y_{1} \ldots y_{n}\right) \in \mathcal{Q}_{x} \times \mathcal{Q}_{y}: x_{j}=y_{k} \text { for some } j, k\right\}
$$

of "collision configurations" (i.e., ones in which an $x$ and a $y$ meet). As shown by Lampart [20], there exists a well-defined, self-adjoint IBC Hamiltonian for this model. This is particularly remarkable insofar as no renormalized Hamiltonian $H_{\infty}$ was known for this case prior to Lampart's work. Moreover, since in this model the formal expression for the interaction Hamiltonian is, instead of (5),

$$
H_{\mathrm{int}}=g \int d^{3} x b^{\dagger}(x)\left(a(x)+a^{\dagger}(x)\right) b(x)
$$

with $b$ being the fermion annihilation operator, the model is also an example in which the Hamiltonian is not quadratic in the creation and annihilation operators.

IBCs have been studied mathematically for several other cases: Lampart and Schmidt [21] proved the existence of an IBC Hamiltonian for a model with moving $x$-particles in 2 dimensions as well as for a model with a different dispersion relation, replacing the Laplace operator $-\frac{\hbar^{2}}{2 m_{y}} \nabla^{2}$ by the pseudo-relativistic 1-particle Hamiltonian $\sqrt{m_{y}^{2}-\nabla^{2}}$, a model for which Nelson [22] could prove the existence of a renormalized Hamiltonian in 1964. IBC models in 1 dimension were studied in $[23,24]$. Early works on the model with $x$ fixed at $\mathbf{0}[17,25]$ used a truncated Fock space with only 2 or 3 sectors.

Profiles other than $\delta^{3}$ for the emission and absorption terms are often desired in connection with relativistic dispersion relations; they lead to conditions that are no longer literally boundary conditions but have been termed "abstract boundary conditions" [26]; the IBC approach has been extended in this direction in [21,27-30].

The considerations of probability transport in Section 2.2 apply literally in the Bohmian picture, in which the particles are attributed trajectories that can begin and end at emission and absorption events, corresponding to jumps in the actual configuration from $\mathcal{Q}^{(n)}$ to $\partial \mathcal{Q}^{(n+1)}$ or vice versa [11,31].

A pedagogical introduction to IBCs can be found in [16]; the behavior of IBCs under time reversal was studied in [18]. Apart from the boundary sets (9) and (59), it is natural 
to also consider other domains with boundaries and, among them, in particular with boundaries of codimension 1 (whereas (9) and (59) have codimension 3). First discussed in [16,31], IBCs on codimension-1 boundaries were systematically studied in [19] for the Laplacian (i.e., for nonrelativistic Hamiltonians) and in [24,32] for the Dirac equation. Lienert and Nickel [24] developed a QFT model in 1-space dimension in which moving $x$-particles emit and absorb other $x \mathrm{~s}, x \rightleftarrows 2 x$, based on the Dirac operator as the free Hamiltonian; they specified an IBC and proved results implying the existence of a welldefined, self-adjoint Hamiltonian.

In 3 space dimensions, in contrast, the free Dirac operator does not allow for a boundary condition on the set of collision configurations (or any subset of codimension 3) and thus not for an IBC [33]. That sounds discouraging at first. However, in nature, the relevant particle reaction is not $x \rightleftarrows 2 x$ but $x \rightleftarrows x+y$, with the $y$-particles being photons, governed not by the Dirac equation but by the Maxwell equation, and this case has not been settled yet. Moreover, despite the impossibility theorem just described, a model in which Dirac particles are emitted and absorbed by a spin- 0 particle fixed at the origin has been proven [33] to possess a well-defined and self-adjoint IBC Hamiltonian if the Dirac particles feel a sufficiently strong $1 / r$ potential (such as a Coulomb or gravitational potential), regardless of whether the potential is attractive or repulsive. It would be of interest to study rigorously whether an IBC Hamiltonian exists for Dirac particles in a general-relativistic gravitational field of a point mass (such as the Reissner-Nordström space-time geometry); preliminary (non-rigorous) considerations in this direction can be found in [34].

\subsection{Interior-Boundary Conditions for the Dirac Equation}

Let me give some explicit examples of what IBCs look like for the Dirac equation. I will stay away from the difficulties and technicalities associated with the situation in which the boundary has codimension 3, a situation that arises in 3 space dimensions when the boundary is the set of configurations in which two particles meet, as in (9) and (59). Instead, I will consider the more elementary example of a boundary of codimension 1 [24,32], which arises either when we consider space dimension 1 or when we study an artificially simplified example of a boundary. Here, we do the latter.

Therefore, consider, as a simple example, the free 1-particle Dirac equation

$$
i \hbar \partial_{t} \psi=-i \hbar \alpha \cdot \nabla \psi+m \beta \psi
$$

in 3 space dimensions in a region $\Omega \subset \mathbb{R}^{3}$ with $2 \mathrm{D}$ boundary surface $\partial \Omega$. For concreteness, let us consider the upper half space $\Omega=\left\{\left(x_{1}, x_{2}, x_{3}\right): x_{3} \geq 0\right\}$ with the $x_{1} x_{2}$-plane as the boundary $\partial \Omega$.

The boundary conditions for the Dirac equation specify two out of the four components of $\psi$ at every boundary point and leave the other two components arbitrary. More precisely, they split the Dirac spin space $S=\mathbb{C}^{4}$ into a direct sum of two 2D subspaces, $S=U \oplus V$; specify the part $\psi_{U}$ of $\psi=\psi_{U}+\psi_{V}$ in $U$; and put no condition on the other part $\psi_{V}$.

For example, let us look at the best known case of a reflecting boundary condition, viz., the boundary condition of the "MIT bag model" of quark confinement [35],

$$
\left(\gamma^{3}-i\right) \psi\left(x_{1}, x_{2}, 0\right)=0
$$

(Here, $i$ means $i$ times the identity matrix.) Since $\gamma^{3}$ is unitarily diagonalizable with eigenvalues $\pm i$ and $2 \mathrm{D}$ eigenspaces, $\gamma^{3}-i$ is $-2 i$ times an orthogonal projection; therefore, this condition says that, at every boundary point, $\psi$ has to lie in the eigenspace with eigenvalue $+i$; put differently, $U$ is the eigenspace with eigenvalue $-i, \psi_{U}$ is prescribed to vanish, $V$ is the eigenspace with eigenvalue $+i$, and no condition is put on $\psi_{V}$.

If the situation that half of the components can be prescribed seems unfamiliar, then it may be useful to note that, in the nonrelativistic case, a boundary condition may involve, 
at every boundary point $x \in \partial \Omega$, the value $\psi(x)$ as well as the normal derivative $\partial_{n} \psi(x)$ to the boundary at $x$. If $\psi$ has $s$ components, then $\left(\psi(x), \partial_{n} \psi(x)\right)$ has $2 s$ components and the boundary condition will usually be of the form

$$
A(x) \psi(x)+B(x) \partial_{n} \psi(x)=0
$$

with $s \times s$ matrices $A$ and $B$. Therefore, the condition consisting of $s$ equations specifies half of the components while putting no condition on the other half (more precisely, it specifies the part of $\left(\psi(x), \partial_{n} \psi(x)\right)$ in an s-dimensional subspace of $\mathbb{C}^{2 s}$, that is, in half of the dimensions, if the juxtaposition of $A$ and $B$ has rank s).

Now, I describe an example of an IBC analogous to (62). For such an example, we need at least two sectors of the wave function and of configuration space. Therefore, let us assume, for simplicity, that there are exactly two sectors: that the configuration space is $\mathcal{Q}=\mathcal{Q}^{(0)} \cup \mathcal{Q}^{(1)}$, where $\mathcal{Q}^{(0)}$ has just one element (the "empty configuration") and $\mathcal{Q}^{(1)}=\Omega$, and that the Hilbert space is $\mathscr{H}=\mathbb{C} \oplus L^{2}\left(\Omega, \mathbb{C}^{4}\right)$. The function $\psi^{(1)}$ obeys the Dirac equation (61) at every point in the half space $\Omega$, while

$$
i \hbar \frac{\partial \psi^{(0)}}{\partial t}=\int_{\mathbb{R}^{2}} d x_{1} d x_{2} N\left(x_{1}, x_{2}\right)^{\dagger} \psi^{(1)}\left(x_{1}, x_{2}, 0\right),
$$

where $N\left(x_{1}, x_{2}\right)$ is a fixed spinor field that is square-integrable and satisfies

$$
N^{\dagger}\left(x_{1}, x_{2}\right) \gamma^{3} \gamma^{0} N\left(x_{1}, x_{2}\right)=0
$$

at every $\left(x_{1}, x_{2}\right) \in \mathbb{R}^{2}$. The IBC reads

$$
\left(\gamma^{3}-i\right) \psi^{(1)}\left(x_{1}, x_{2}, 0\right)=-\frac{i}{\hbar}\left(\gamma^{3}-i\right) \gamma^{3} \gamma^{0} N\left(x_{1}, x_{2}\right) \psi^{(0)} .
$$

Again, the boundary condition prescribes the part of $\psi^{(1)}\left(x_{1}, x_{2}, 0\right)$ in $U$ while putting no condition on its part in $V$, and again, the condition couples the two sectors of $\psi$. The spinor $N$ remains arbitrary as a parameter of the model. See [32] for a proof of self-adjointness of the Hamiltonian defined by (61) and (64) on a domain of wave functions satisfying the IBC (66) (in a slightly different setting with compact $\partial \Omega$ ) and a discussion of further variants of this IBC.

\section{Quantum Electrodynamics}

IBCs have not yet been made to work for QED, but I want to describe reasons for thinking that they can and, more generally, for thinking that wave functions in the particleposition representation can be helpful in QED. To this end, I first need to talk about multi-time wave functions.

\subsection{Multi-Time Wave Functions}

Wave functions in the particle-position representation are functions of the positions of the particles, $\psi=\psi\left(x_{1} \ldots x_{n}\right)$. In the nonrelativistic picture, this wave function evolves with time. In the relativistic picture, it does not seem appropriate any more to consider several space points at the same time coordinate, as that situation would depend on the choice of Lorentz frame. The natural relativistic version would be to consider wave functions that are functions of several space-time points, $\phi=\phi\left(x_{1} \ldots x_{n}\right)$ with each $x_{j} \in \mathbb{R}^{4}$ or $x_{j} \in \mathscr{M}$ with $\mathscr{M}$ the space-time manifold. We usually take $\phi$ to be defined on the set of space-like space-time configurations

$$
\mathscr{S}=\bigcup_{n=0}^{\infty} \mathscr{S}_{n}=\bigcup_{n=0}^{\infty}\left\{\left(x_{1} \ldots x_{n}\right) \in \mathscr{M}^{n}: x_{j}=x_{k} \text { or } x_{j} \times x_{k} \text { for all } j, k\right\},
$$


where $x \times y$ means that $x$ and $y$ are space-like separated. Since $\phi$ involves several time variables $x_{1}^{0}, \ldots, x_{n}^{0}$, it is called a multi-time wave function. This concept, a covariant expression of the state vector, was suggested early on in the history of quantum physics by Eddington [36] and Dirac [37]; see [38] for a comprehensive discussion and further references.

Since $\phi^{(n)}=\left.\phi\right|_{\mathscr{S}_{n}}$ depends on $n$ time variables, its time evolution, if governed by PDEs, needs $n$ equations, one for each time variable, as in

$$
i \hbar \frac{\partial \phi^{(n)}}{\partial x_{j}^{0}}=H_{j}^{(n)} \phi ，
$$

where $H_{j}^{(n)}$ is an operator called the $j$ th partial Hamiltonian; it can roughly be thought of as collecting the terms in the Hamiltonian pertaining to $x_{j}$, and we have set $c=1$. Such a system of equations can be inconsistent, but consistency has been verified for relativistic versions of the Lee model analogous to (2) $[24,39,40]$. An upshot at this point is that the particle-position representation fits nicely together with Lorentz invariance.

A version of the Born rule appropriate for $\phi$ is the curved Born rule [41,42]: If detectors are placed along a (possibly curved) Cauchy surface $\Sigma$ (i.e., a space-like 3-surface) in $\mathscr{M}$, then the probability density (relative to the Riemannian volume measure) of finding the configuration $\left(x_{1}, \ldots, x_{n}\right) \in \Sigma^{n}$ is given by

$$
\rho\left(x_{1} \ldots x_{n}\right)=\left|\phi^{(n)}\left(x_{1} \ldots x_{n}\right)\right|^{2},
$$

with $|\cdot|^{2}$ suitably understood, such as,

$$
\left|\phi\left(x_{1} \ldots x_{n}\right)\right|^{2}=\bar{\phi}\left(x_{1} \ldots x_{n}\right)\left[\gamma^{\mu_{1}} n_{\mu_{1}}\left(x_{1}\right) \otimes \cdots \otimes \gamma^{\mu_{n}} n_{\mu_{n}}\left(x_{n}\right)\right] \phi\left(x_{1} \ldots x_{n}\right)
$$

for Dirac wave functions with $n_{\mu}(x)$ being the future unit normal vector to $\Sigma$ at $x$.

\subsection{Landau and Peierls}

In 1930, Landau and Peierls [43] wrote down a version of QED (simplified without positrons) in the particle-position representation. Here, I reproduce their equations in a multi-time form developed recently [44]. The wave function depends on the space-time points $x_{1}, \ldots, x_{m}$ of a variable number $m$ of electrons, as well as the space-time points $y_{1}, \ldots, y_{n}$ of a variable number $n$ of photons, $\phi=\phi\left(x_{1} \ldots x_{m}, y_{1} \ldots y_{n}\right)$. We take it that the wave function of a single electron is a Dirac wave function with 4 complex components labeled by the index $s=1 . . .4$ and that the wave function of a single photon is a complexified Maxwell field represented by its vector potential $A_{\mu}$, also with 4 complex components but now labeled by the index $\mu=0 . . .3$. Thus,

$$
\phi=\phi_{s_{1} \ldots s_{m} \mu_{1} \ldots \mu_{n}}^{(m, n)}\left(x_{1} \ldots x_{m}, y_{1} \ldots y_{n}\right) .
$$

A Lorentz transformation acts on each $x_{j}$ and each $y_{k}$ in the usual way, on each index $\mu_{k}$ as on the index of a world vector, and on each index $s_{j}$ as on the index of a Dirac spinor; thus, the action on $\phi^{(m, n)}$ is the product of representations of the proper Lorentz group. The domain of definition of $\phi$ is the set of space-like configurations of $x$ s and $y$ s, i.e., where any two of $x_{1} \ldots x_{m}, y_{1} \ldots y_{n}$ are either space-like or equal. $\phi$ is symmetric against permutation of the photons (permuting the space-time points $y_{k}$ and the indices $\mu_{k}$ in the same way) and anti-symmetric against permutation of the electrons (of $x_{j}$ and $s_{j}$ alike). Let $e$ and $m_{x}$ denote the charge and the mass of the electron. The time evolution is governed by 3 equations, (72), (73), and (74) below. For now, we will not worry about UV divergence and simply write down Dirac delta distributions. 
The equation in $x_{j}$ is the Dirac equation with an additional term:

$$
\left(i \gamma_{j}^{\mu} \partial_{x_{j}, \mu}-m_{x}\right) \phi^{(m, n)}\left(x_{1} \ldots x_{m}, y_{1} \ldots y_{n}\right)=e \sqrt{n+1} \gamma_{j}^{\rho} \phi_{\mu_{n+1}=\rho}^{(m, n+1)}\left(x_{1} \ldots x_{m}, y_{1} \ldots y_{n}, x_{j}\right)
$$

where $\gamma_{j}^{\mu}$ means $\gamma^{\mu}$ acting on the index $s_{j}$ and most indices have not been made explicit.

The equation in $y_{k}$ is the Maxwell equation with a source term:

$$
\begin{aligned}
2 \partial_{y_{k}}^{\mu} \partial_{y_{k},[\mu} \phi_{\left.\mu_{k}=v\right]}^{(m, n)}\left(x_{1} \ldots x_{m}, y_{1} \ldots y_{n}\right) & = \\
& \frac{4 \pi e}{\sqrt{n}} \sum_{j=1}^{m} \delta_{\mu}^{3}\left(y_{k}-x_{j}\right) \gamma_{j}^{\mu} \gamma_{j v} \phi_{\overline{\mu_{k}}}^{(m, n-1)}\left(x_{1} \ldots x_{m}, y_{1} \ldots y_{k-1}, y_{k+1} \ldots y_{n}\right) .
\end{aligned}
$$

where $[\mu v]$ means anti-symmetrization in the index pair as in $S_{[\mu v]}=\frac{1}{2}\left(S_{\mu \nu}-S_{\nu \mu}\right)$; the 3-dimensional Dirac delta distribution wears a space-time index $\mu$ because it is a vector field in the 4 dimensional set $\left\{x \in \mathbb{R}^{4}: x=0\right.$ or $\left.x \times 0\right\}$ (corresponding to a 3-form that can be integrated over any space-like surface through $0 \in \mathbb{R}^{4}[44]$ ); and $\widehat{\mu_{k}}$ means that the index $\mu_{k}$ is omitted.

The third equation is a gauge condition that is actually not Lorentz invariant,

$$
\sum_{\mu_{k}=1}^{3} \partial_{y_{k}}^{\mu_{k}} \phi_{\mu_{k}}^{(m, n)}\left(x_{1} \ldots x_{m}, y_{1} \ldots y_{n}\right)=0
$$

where $\mu_{k}=0$ is omitted. Again, most indices are not made explicit. This equation is analogous to the Coulomb gauge condition

$$
\sum_{\mu=1}^{3} \partial^{\mu} A_{\mu}=0
$$

To understand the Equations (72)-(74), let us compare them to 1-particle equations. The 1-particle Dirac equation in an external electromagnetic field with vector potential $A_{\mu}(x)$ reads

$$
\left(i \gamma^{\mu} \partial_{\mu}-m_{x}\right) \phi(x)=e \gamma^{\rho} A_{\rho}(x) \phi(x) .
$$

Equation (72) has the same structure with three differences: it applies to $x_{j}$ in a multi-time function, the factor $\sqrt{n+1}$ in a sense compensates overcounting due to symmetrization, and most importantly, the external field $A_{\mu}$ has been replaced by the wave function of the $n+1$-st photon. If $\phi^{(m, n+1)}$ factorized according to

$$
\phi_{\mu_{n+1}}^{(m, n+1)}\left(x_{1} \ldots x_{m}, y_{1} \ldots y_{n+1}\right)=A_{\mu_{n+1}}\left(y_{n+1}\right) \phi^{(m, n)}\left(x_{1} \ldots x_{m}, y_{1} \ldots y_{n}\right),
$$

then (72) would reduce exactly to (76) applied to $x_{j}$ (except for the factor $\sqrt{n+1}$ that stems from our normalization convention). In other words, (72) is essentially the Dirac equation in which the vector potential is provided by the wave function of the next photon.

The 1-photon equation, i.e., the complex Maxwell equation, with source term $J_{v}(y)$ reads

$$
2 \partial^{\mu} \partial_{[\mu} A_{v]}(y)=4 \pi J_{v}(y),
$$

which is a reformulation of the well-known form $\partial^{\mu} F_{\mu \nu}=4 \pi J_{v}$ using $F_{\mu v}=2 \partial_{[\mu} A_{v]}$. Equation (73) has the same structure, up to the factor $1 / \sqrt{n}$ that compensates overcounting, with $\phi_{\mu_{k}}$ playing the role of $A_{\mu}, y_{k}$ playing the role of $y$, and the source term given by

$$
J_{\nu}(y)=e \sum_{j=1}^{m} \delta_{\mu}^{3}\left(y-x_{j}\right) \gamma_{j}^{\mu} \gamma_{j, v} \phi_{\widehat{\mu_{k}}}^{(m, n-1)}\left(\widehat{y_{k}}\right)
$$


This term is indeed concentrated on the locations $x_{1}, \ldots, x_{m}$ of the electrons; moreover, when the term is integrated over a horizontal surface with respect to the chosen Lorentz frame, the Dirac delta contributes only for $\mu=0$, so $\gamma^{\mu} \gamma_{v}$ becomes $\gamma^{0} \gamma_{v}=\alpha_{v}$, which is the matrix associated with the current in the wave function $\phi$.

What I am getting at is that (72) and (73) are very natural equations; they are basically the Dirac and Maxwell equations applied to particular variables of $\phi$, with natural expressions inserted for the vector potential and the source term, given that the photon aspect of $\phi$ is analogous to $A_{\mu}$ and the electron aspect of $\phi$ should be the source for the Maxwell equation. Moreover, the terms coupling the time derivatives of $\phi^{(m, n)}$ to the neighboring sectors $\phi^{(m, n+1)}$ and $\phi^{(m, n-1)}$ lead, in every Lorentz frame, to an evolution similar to (2), with photons emitted and absorbed by electrons, as they should be. Obviously, (72) and (73) are Lorentz invariant. The question of consistency of (72)-(74) is subtle and needs further investigation [44]. If the system is consistent, then we can say that QED (in this simplified form without positrons) fits very nicely into the framework of the particle-position representation.

Here is what (73) has to do with IBCs. It is well known that part of the Maxwell equations determine the time derivatives while another part is a constraint. In any fixed Lorentz frame, the constraint equations contained in (78) read

$$
\nabla \cdot \boldsymbol{B}=0, \quad \nabla \cdot \boldsymbol{E}=-4 \pi J_{0}
$$

with $\boldsymbol{B}^{i}=\varepsilon^{i j k} F_{j k}$ and $\boldsymbol{E}_{i}=F_{0 i}$. If $J_{0}(\boldsymbol{y})=e \delta^{3}(\boldsymbol{y})$, then integration of the second equation over a ball $B_{r}(\mathbf{0})$ of radius $r$ yields, by the Ostrogradski-Gauss integral theorem,

$$
-4 \pi e=\int_{B_{r}(\mathbf{0})} d^{3} \boldsymbol{y}(-4 \pi e) \delta^{3}(\boldsymbol{y})=\int_{B_{r}(\mathbf{0})} d^{3} \boldsymbol{y} \nabla \cdot \boldsymbol{E}(\boldsymbol{y})=\int_{\mathbb{S}^{2}} d^{2} \boldsymbol{\omega} r^{2} \boldsymbol{\omega} \cdot \boldsymbol{E}(r \boldsymbol{\omega}) .
$$

Suppose that, in the limit $r \searrow 0$, the radial component $E_{r}=\boldsymbol{\omega} \cdot \boldsymbol{E}$ of $\boldsymbol{E}$ does not depend on the direction $\omega$ any more. Then, (81) yields that

$$
\lim _{r \searrow 0} r^{2} E_{r}(r \omega)=-e .
$$

For the wave function $\phi$, this implies the following. For simplicity, consider a single $x$ particle fixed at the origin. Then (because $\gamma^{0} \gamma^{0}=1$ ), when all time coordinates are set to 0 , the second equation of (80) becomes

$$
\lim _{r \searrow 0} r^{2} E_{r}\left(\boldsymbol{x}=\mathbf{0}, \boldsymbol{y}_{1} \ldots y_{n-1}, r \boldsymbol{\omega}\right)=-e \phi_{\widehat{\mu}_{n}}^{(1, n-1)}\left(\widehat{y_{n}}\right)
$$

with $E_{r}$ being the appropriate $\boldsymbol{y}_{n}$-derivative of $\phi^{(1, n)}$,

$$
E_{r}\left(\boldsymbol{x}=\mathbf{0}, \boldsymbol{y}_{1} \ldots y_{n-1}, r \boldsymbol{\omega}\right)=\sum_{i=1}^{3} \omega_{i}\left(\partial_{y_{n}, 0} \phi_{\mu_{n}=i}^{(1, n)}-\partial_{y_{n, i}} \phi_{\mu_{n}=0}^{(1, n)}\right)\left(\boldsymbol{x}=\mathbf{0}, \boldsymbol{y}_{1} \ldots y_{n-1}, r \boldsymbol{\omega}\right)
$$

Equation (83) is an IBC: It is a relation between values (or asymptotics) of $\phi$ (or its derivatives) at $r=0$ and values of $\phi$ at the configuration with one photon removed, i.e., at the configuration related through the absorption of a photon. Note also that the IBC is gauge invariant, as it is expressed in terms of components of $F_{\mu v}$, not $A_{\mu}$. (On earlier occasions [4,10], I had written that Landau and Peierls had the first IBC. Thinking again about it, I find that statement too strong, as they wrote their constraint condition with delta distributions, not with boundary conditions.)

I see two main open problems with the approach of Landau and Peierls (which might be connected): the status of the gauge condition and the Born rule for photons. What is puzzling about the gauge condition (74) is that, while Landau and Peierls thought of it as a matter of convenience, it seems that we cannot simply dispense with any gauge 
condition at all without obtaining too many solutions to the system comprising only (72) and (73) [44]. It also seems that the empirical predictions of the theory (even though it is a simplified model that should not be expected to be fully empirically adequate) will depend on the choice of gauge condition, as if there was one "correct" condition. However, which one would that be, and why? The other problem deserves a section of its own.

\subsection{The Problem of Born's Rule for Photons}

While it is widely agreed upon that the quantum state of a single free photon is mathematically equivalent to a (complexified) Maxwell field $F_{\mu v}$, there is no agreed-upon answer to what the Born rule for a single photon would say, i.e., for how to compute the probability density $\rho$ in the position space from the wave function $F_{\mu \nu}$. For comparison, for a Dirac wave function $\psi: \mathbb{R}^{4} \rightarrow \mathbb{C}^{4}$, the probability density $\rho_{\mathrm{D}}$ or, equivalently, the time component $j_{\mathrm{D}}^{0}$ of the probability current 4 -vector $j_{\mathrm{D}}^{\mu}$ is determined by

$$
j_{\mathrm{D}}^{\mu}(x)=\bar{\psi}(x) \gamma^{\mu} \psi(x) .
$$

To be sure, there is a convincing way of computing the photon probability current $j^{\mu}$ provided that the photon wave function (or, equivalently, Maxwell field) $F_{\mu v}$ is a plane wave or at least a local plane wave (i.e., a function such that every point has a small neighborhood on which the function is to a good degree of approximation a plane wave): Suppose

$$
A_{\mu}(x)=a_{\mu} e^{i k_{\lambda} x^{\lambda}}, \quad F_{\mu v}(x)=2 i a_{[\mu} k_{v]} e^{i k_{\lambda} x^{\lambda}}
$$

with future-light-like $k^{\mu}$ and $k^{\mu} a_{\mu}=0$ to fulfill the free Maxwell equation $\partial^{\mu} F_{\mu \nu}=0$. If many photons have the same wave function, a classical regime with electromagnetic field $F_{\mu \nu}$ (or something closely related) should apply. Classically, the energy-momentum density is given, up to constant factors, by the tensor field

$$
T_{\mu \nu}=\operatorname{Re}\left[F_{\mu \lambda}^{*} F_{v}^{\lambda}\right]-\frac{1}{4} g_{\mu \nu} F_{\lambda \rho}^{*} F^{\lambda \rho},
$$

which for the plane wave (86) amounts to

$$
T_{\mu \nu}=a_{\lambda}^{*} a^{\lambda} k_{\mu} k_{v} .
$$

If each photon has momentum $\hbar k^{\mu}$ according to the de Broglie relation and if photon number density is proportional to the probability density $j^{v}$ for each photon, then the energy-momentum density should be

$$
T_{\mu \nu}=\hbar k_{\mu} j_{v}
$$

up to a constant factor. Therefore, we can obtain $j^{\mu}$ by comparison with (88),

$$
j^{\mu}=a_{\lambda}^{*} a^{\lambda} k^{\mu}
$$

up to a constant factor.

However, not every Maxwell field is a local plane wave (even if perhaps, in most present-day experiments, it is a local plane wave); therefore, the question remains, which law determines $j^{\mu}$ in general? Of the desired law, I would expect these properties:

1. The expression is quadratic in $A_{\mu}$ and its derivatives.

2. The expression is local, i.e., $j^{\nu}(x)$ depends only on $A_{\mu}$ and its derivatives at $x$.

3. $j^{\mu}$ is future-time-like or -light-like.

4. $\partial_{\mu} j^{\mu}=0$ if $A_{\mu}$ obeys the free Maxwell equations.

5. For a plane wave, $j^{\mu}$ agrees with (90) up to a constant factor.

6. No choices need to be made, i.e., if the law requires a special gauge or Lorentz frame, then it also specifies this gauge or Lorentz frame.

7. The law can be generalized to curved space-time. 
If the law involves $A_{\mu}$, then it might also involve a gauge condition that selects a particular gauge and replaces (74), thereby also solving the problem mentioned at the end of Section 3.2. Several proposals for $j^{\mu}$ have been made (e.g., [43,45,46]), but none of them satisfies all of the properties above, so I am hesitant to accept any of them, even though some of them may be useful approximations. It seems to me that the correct answer to the question has not been found yet, although it seems to be a very basic question.

\section{Detection Time and Boundary Conditions}

Another boundary condition on wave functions deserves to be mentioned: an absorbing boundary condition used for formulating Born's rule on a time-like 3-surface $\Sigma$. Consider a 2-surface $\sigma$ in 3 space, and suppose that detectors are placed along $\sigma$ waiting for a quantum particle to arrive. When and where on $\sigma$ will the particle be detected? In other words, where on the time-like 3-surface $\Sigma=[0, \infty) \times \sigma$ in 4-space-time will it be detected? (Assuming the initial conditions are set up on the surface $\left\{x^{0}=0\right\}$.) The problem of computing the probability distribution of the detection point $X \in \Sigma$ is known as the time-of-arrival problem, although it would perhaps be more accurately called the time-of-detection problem.

Let me focus again on the nonrelativistic case, and let us assume that $\sigma$ is the boundary of a 3-region $\Omega$; for simplicity, I ignore the possibility that no detector ever clicks. General measurement theory yields that there is a POVM (positive-operator-valued measure) $E(\cdot)$ on $\Sigma$ such that the probability distribution of $X$ is given by

$$
\mathbb{P}(X \in B)=\left\langle\psi_{0}|E(B)| \psi_{0}\right\rangle
$$

for every subset $B$ of $\Sigma$ and every initial wave function $\psi_{0}$, but it does not tell us in an accessible way which operator $E(B)$ is.

However, heuristic considerations [47] suggest the following simple rule for calculating $\mathbb{P}(X \in B)$ for idealized detectors, called the absorbing boundary rule [47,48], and this is what I want to explain in this section. In $L^{2}(\Omega)$, solve the Schrödinger equation

$$
i \hbar \frac{\partial \psi}{\partial t}=-\frac{\hbar^{2}}{2 m} \nabla^{2} \psi(x)+V(x) \psi(x)
$$

subject to the boundary condition

$$
\boldsymbol{n}(\boldsymbol{x}) \cdot \nabla \psi(\boldsymbol{x})=i \kappa \psi(\boldsymbol{x}) \text { for all } x \in \sigma=\partial \Omega
$$

with $\boldsymbol{n}(\boldsymbol{x})$ the unit normal vector to $\sigma$ at $x$ and $\kappa$ a given constant such that $\hbar^{2} \kappa^{2} / 2 m$ is the energy of maximal efficiency of the detectors. Then,

$$
\mathbb{P}(X \in B)=\int_{B} d t d^{2} x \boldsymbol{n}(\boldsymbol{x}) \cdot \boldsymbol{j}(\boldsymbol{x}, t)
$$

with $j$ the usual probability current,

$$
j=\frac{\hbar}{m} \operatorname{Im}\left[\psi^{*} \nabla \psi\right] .
$$

Due to the boundary condition (93), the integrand in (94) is actually nonnegative, as a probability density should be:

$$
\begin{aligned}
\boldsymbol{n}(\boldsymbol{x}) \cdot \boldsymbol{j}(\boldsymbol{x}, t) & =\frac{\hbar}{m} \operatorname{Im}\left[\psi(\boldsymbol{x}, t)^{*} \boldsymbol{n}(\boldsymbol{x}) \cdot \nabla \psi(\boldsymbol{x}, t)\right] \\
& =\frac{\hbar}{m} \operatorname{Im}\left[\psi(\boldsymbol{x}, t)^{*} i \kappa \psi(\boldsymbol{x}, t)\right] \\
& =\frac{\kappa \hbar}{m}|\psi(\boldsymbol{x}, t)|^{2} \geq 0 .
\end{aligned}
$$


This means that, on the boundary, the current always points outwards, not inwards, so that the particle can cross the boundary only outwards, not inwards. It also implies that the $L^{2}$ norm of $\psi_{t}$ is not constant but shrinks with time,

$$
\frac{d}{d t}\left\|\psi_{t}\right\|^{2}=\frac{d}{d t} \int_{\Omega} d^{3} x|\psi(x, t)|^{2} \leq 0 .
$$

Thus, the time evolution is not unitary (but is what is called a contraction semi-group). It is known [49] that the time evolution is well-defined, i.e., that Schrödinger's equation (92) with the boundary condition (93) possesses a unique solution for every $\psi_{0} \in L^{2}(\Omega)$. This fact reflects the intuitive idea that any part of the wave function $\Psi$ of the object $x$ and detectors together that has crossed $\sigma$ in the $x$ variable has decohered, i.e., cannot any longer form a coherent superposition with the part that has not crossed $\sigma$ yet.

That is, the boundary condition here models the effect of the presence of the detector on the wave function in $\Omega$ by precluding backflow of any part of the wave function from outside of $\Omega$. It can be shown $[47,49]$ that this rule indeed defines a POVM $E(\cdot)$. It would be of interest to study whether the absorbing boundary rule can be derived from a microscopic quantum-mechanical model of the detectors.

There are several parallels between IBCs and the absorbing boundary condition (ABC) (93). Both are conditions on the wave function. Both are perhaps best understood in terms of their effects on probability transport. Both have been studied only rather recently. Moreover, while the IBC provides a way around the UV divergence problem, the ABC provides a way around the quantum Zeno effect that was long thought to make any mathematically clean concept of a "hard" detector, i.e., one that detects the particle as soon as it reaches the surface $\sigma$, impossible.

\section{Conclusions}

I have given a brief overview of recent research about two kinds of boundary conditions: interior-boundary conditions and absorbing boundary conditions. Both are boundary conditions on wave functions in the particle-position representation, the former for the time evolution with particle creation at point sources and the latter for the time evolution in the presence of hard detectors. As IBCs provide a way out of the UV divergence problem often encountered in connection with particle creation, they are of interest in QFT. They have been proven to work in the nonrelativistic case, while the relativistic case remains a field for future research. I have outlined some aspects of how this research might proceed.

Funding: This work received no external funding.

Conflicts of Interest: The author declares no conflict of interest.

\section{References}

1. Moshinsky, M. Boundary Conditions for the Description of Nuclear Reactions. Phys. Rev. 1951, 81, 347-352. [CrossRef]

2. Moshinsky, M. Boundary Conditions and Time-Dependent States. Phys. Rev. 1951, 84, 525-532. [CrossRef]

3. Moshinsky, M. Quantum Mechanics in Fock Space. Phys. Rev. 1951, 84, 533. [CrossRef]

4. Teufel, S.; Tumulka, R. Hamiltonians Without Ultraviolet Divergence for Quantum Field Theories. Quantum Stud. Math. Found. 2021, 8, 17-35. [CrossRef]

5. Lee, T.D. Some Special Examples in Renormalizable Field Theory. Phys. Rev. 1954, 95, 1329-1334. [CrossRef]

6. Schweber, S. An Introduction To Relativistic Quantum Field Theory; Harper and Row: New York, NY, USA, 1961.

7. Dereziński, J. Van Hove Hamiltonians-Exactly Solvable Models of the Infrared and Ultraviolet Problem. Ann. Henri Poincaré 2003, 4, 713-738. [CrossRef]

8. Van Hove, L. Les difficultés de divergences pour un modèle particulier de champ quantifié. Physica 1952, 18, 145-159. [CrossRef]

9. Oppenheimer, J.R. Note on the Theory of the Interaction of Field and Matter. Phys. Rev. 1930, 35, 461-477. [CrossRef]

10. Lampart, J.; Schmidt, J.; Teufel, S.; Tumulka, R. Particle Creation at a Point Source by Means of Interior-Boundary Conditions. Math. Phys. Anal. Geom. 2018, 21, 12. [CrossRef]

11. Dürr, D.; Goldstein, S.; Teufel, S.; Tumulka, R.; Zanghì, N. Bohmian Trajectories for Hamiltonians with Interior-Boundary Conditions. J. Stat. Phys. 2020, 180, 34-73. [CrossRef]

12. Epstein, H.; Glaser, V. The role of locality in perturbation theory. Ann. L'Institut Henri Poincaré A Phys. Théorique 1973, 19, 211-295. 
13. Scharf, G. Finite Quantum Electrodynamics, 2nd ed.; Springer: Berlin, Germany, 1995.

14. Dell'Antonio, G.; Figari, R.; Teta, A. A brief review on point interactions. In Inverse Problems and Imaging; Bonilla, L.L., Ed.; Lecture Notes in Mathematics 1943; Springer: Berlin, Germany, 2008; pp. 171-189.

15. Bethe, H.; Peierls, R. Quantum Theory of the Diplon. Proc. R. Soc. Lond. A 1935, 148, 146-156.

16. Teufel, S.; Tumulka, R. Avoiding Ultraviolet Divergence by Means of Interior-Boundary Conditions. In Quantum Mathematical Physics-A Bridge between Mathematics and Physics; Finster, F., Kleiner, J., Röken, C., Tolksdorf, J., Eds.; Birkhäuser: Basel, Switzerland, 2016; pp. 293-311.

17. Yafaev, D.R. On a zero-range interaction of a quantum particle with the vacuum. J. Phys. A Math. Gen. 1992, 25, 963-978. [CrossRef]

18. Schmidt, J.; Tumulka, R. Complex Charges, Time Reversal Asymmetry, and Interior-Boundary Conditions in Quantum Field Theory. J. Phys. A Math. Theor. 2019, 52, 115301. [CrossRef]

19. Tumulka, R. Interior-Boundary Conditions for Schrödinger Operators on Codimension-1 Boundaries. J. Phys. A Math. Theor. 2020, 53, 155201. [CrossRef]

20. Lampart, J. A nonrelativistic quantum field theory with point interactions in three dimensions. Ann. Henri Poincaré 2019, 20, 3509-3541. [CrossRef]

21. Lampart, J.; Schmidt, J. On Nelson-type Hamiltonians and abstract boundary conditions. Commun. Math. Phys. 2019, 376, 629-663. [CrossRef]

22. Nelson, E. Interaction of nonrelativistic particles with a quantized scalar field. J. Math. Phys. 1964, 5, 1190-1197. [CrossRef]

23. Keppeler, S.; Sieber, M. Particle creation and annihilation at interior boundaries: One-dimensional models. J. Phys. A Math. Theor. 2016, 49, 125204. [CrossRef]

24. Lienert, M.; Nickel, L. Multi-time formulation of particle creation and annihilation via interior-boundary conditions. Rev. Math Phys. 2020, 32, 2050004. [CrossRef]

25. Thomas, L.E. Multiparticle Schrödinger Hamiltonians with point interactions. Phys. Rev. D 1984, 30, 1233-1237. [CrossRef]

26. Behrndt, J.; Micheler, T. Elliptic differential operators on Lipschitz domains and abstract boundary value problems. J. Funct. Anal. 2014, 267, 3657-3709. [CrossRef]

27. Lampart, J. The Renormalised Bogoliubov-Fröhlich Hamiltonian. J. Math. Phys. 2020, 61, 101902. [CrossRef]

28. Posilicano, A. On the self-adjointness of $H+A^{*}+$ A. Math. Phys. Anal. Geom. 2020, 23, 37. [CrossRef]

29. Schmidt, J. On a Direct Description of Pseudorelativistic Nelson Hamiltonians. J. Math. Phys. 2019, 60, 102303. [CrossRef]

30. Schmidt, J. The Massless Nelson Hamiltonian and its Domain. In Mathematical Challenges of Zero-Range Physics; Michelangeli, A., Ed.; Springer: Berlin, Germany, 2021; pp. 57-80.

31. Georgii, H.-O.; Tumulka, R. Some Jump Processes in Quantum Field Theory. In Interacting Stochastic Systems; Deuschel, J.-D., Greven, A., Eds.; Springer: Berlin, Germany, 2004; pp. 55-73.

32. Schmidt, J.; Teufel, S.; Tumulka, R. Interior-Boundary Conditions for Many-Body Dirac Operators and Codimension-1 Boundaries. J. Phys. A Math. Theor. 2019, 52, 295202. [CrossRef]

33. Henheik, J.; Tumulka, R. Interior-Boundary Conditions for the Dirac Equation at Point Sources in 3 Dimensions. arXiv 2020, arXiv:2006.16755.

34. Tumulka, R. Bohmian Mechanics at Space-Time Singularities. I. Timelike Singularities. J. Geom. Phys. 2019, 145, 103478. [CrossRef]

35. Chodos, A.; Jaffe, R.L.; Johnson, K.; Thorn, C.B.; Weisskopf, V.F. New extended model of hadrons. Phys. Rev. D 1974, 9 , 3471. [CrossRef]

36. Eddington, A.S. The charge of an electron. Proc. R. Soc. A 1929, 122, 358-369. [CrossRef]

37. Dirac, P.A.M. Relativistic quantum mechanics. Proc. R. Soc. Lond. A 1932, 136, 453-464.

38. Lienert, M.; Petrat, S.; Tumulka, R. Multi-time Wave Functions: An Introduction; Springer: Heidelberg, Germany, 2020.

39. Lill, S.; Nickel, L.; Tumulka, R. Consistency Proof for Multi-Time Schrödinger Equations with Particle Creation and Ultraviolet Cut-Off. Ann. Henri Poincaré 2021, in press.

40. Petrat, S.; Tumulka, R. Multi-Time Wave Functions for Quantum Field Theory. Ann. Phys. 2014, 345, 17-54. [CrossRef]

41. Lienert, M.; Tumulka, R. Born's rule for arbitrary Cauchy surfaces. Lett. Math. Phys. 2020, 110, 753-804. [CrossRef]

42. Lill, S.; Tumulka, R. Another proof of Born's rule for arbitrary Cauchy surfaces. 2021, in preparation.

43. Landau, L.; Peierls, R. Quantenelektrodynamik im Konfigurationsraum. Z. Phys. 1930, 62, 188-200. English translation: Quantum electrodynamics in configuration space. In Selected Scientific Papers of Sir Rudolf Peierls With Commentary; Dalitz, R.H., Peierls, R., Eds.; World Scientific: Singapore, 1997; pp. 71-82. [CrossRef]

44. Lienert, M.; Tumulka, R. Multi-Time Version of the Landau-Peierls Formulation of Quantum Electrodynamics. 2021, in press.

45. Bialynicki-Birula, I. On the Wave Function of the Photon. Acta Phys. Pol. 1994, 86, 97-116. [CrossRef]

46. Kiessling, M.K.-H.; Tahvildar-Zadeh, A.S. On the Quantum-Mechanics of a Single Photon. J. Math. Phys. 2018, 59, 112302. [CrossRef]

47. Tumulka, R. Distribution of the Time at Which an Ideal Detector Clicks. arXiv 2016, arXiv:1601.03715.

48. Werner, R. Arrival time observables in quantum mechanics. Ann. L'Institute Henri Poincaré Sect. A 1987, 47, 429-449.

49. Teufel, S.; Tumulka, R. Existence of Schrödinger Evolution with Absorbing Boundary Condition. arXiv 2019, arXiv:1912.12057. 